\title{
Aspiration Sclerotherapy: A Simple Endoscopic Modification for Sclerotherapy of Esophageal Varices
}

Sclerotherapy techniques have been modified considerably since Crafoord and Frenckner's initial report (1). With the development of fiberoptic endoscopy, sclerotherapy was used intensively for the treatment of variceal bleeding. Bleeding from unintended puncture of the sclerosing needle, which sometimes occurs because of peristalsis and vomiting, makes it difficult to complete the sclerotherapy. It is also difficult for most endoscopists to give an injection exactly into the intended target 


\section{S58 Unusual Cases and Technical Notes}

side either intravariceally or paravariceally. In our clinic we have made a simple modification to the sclerotherapy procedure. We combine aspiration and sclerotherapy. We use a banding device to suction the varix as in the ligation procedure. The aspiration of the varix or the esophageal wall or both, provides a fixed view of the intended target side. The fixing of the target side means that an appropriate amount of the sclerosing agent can be used without it escaping from the varix. Furthermore, the presence of a banding device at the front of endoscope ensures a safe distance to see and manipulate the injection needle (Figure 1). This distance helps the endoscopist to avoid the accidental insertion of needle. After making the initial diagnostic evaluation of the esophagus and varices and deciding where to inject, one can aspirate the target, then inject the sclerosing agent (Figure 2). The only disadvantages of the procedure may be the narrowing of the vision as well as the ligation, but we solved this problem by using a transparent banding device. Up to now we have applied this method in 12 patients. In none of them did we accidentally insert a needle and cause excessive bleeding from the injection site.

Our procedure is a modification of a previously described method of combination of ligation and sclerotherapy at the same session $(2,3)$. We use a transparent banding device for sclerotherapy just to accomplish the aspiration. In this procedure, the endoscopist does not need to reload the device and so repeated insertion of endoscope is avoided. It is also far cheaper than multiple banding. We recommend our modification in any patients, particularly those expected to have excessive esophagus motility or to vomit during sclerotherapy.

\section{Y. Akcan, S. Arslan}

Section of Gastroenterology,

Dept. of Medicine, Hacettepe University

Medical School, Ankara, Turkey

\section{References}

1. Crafoord C, Frenckner P. New surgical treatment of varicous veins of the oesophagus. Acta Otolaryngol 1939; 27: $422-5$.

2. Nishikawa Y, Hosokawa Y, Doi T, et al. Simultaneous combination of endoscopic sclerotherapy and endoscopic ligation for esophageal varices (EISL) abstract. Gastroenterology 1996; 110: Al 206.

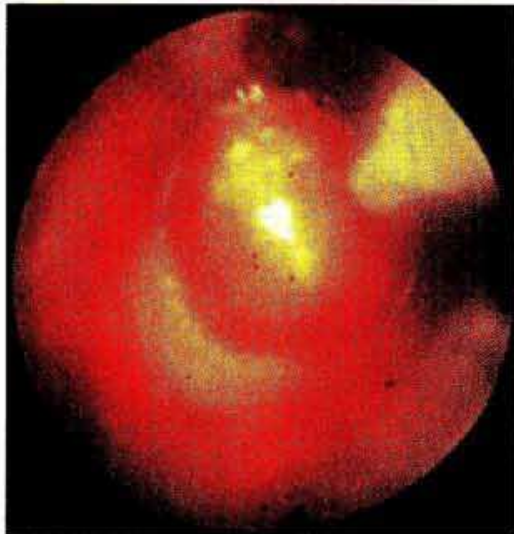

Figure 1: The aspirated varix is fixed and the needle is ready.

3. Rösch T, Allescher HD. Digestive Diseases Week Report 1996, San Francisco, 19-22 May 1996. Review of Endoscopic Abstracts. Endoscopy 1996; $28: 578-625$.

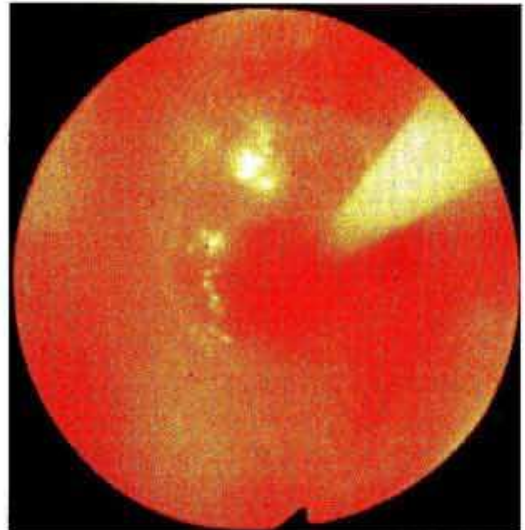

Figure 2: The targeted varix is injected safely without any sign of bleeding.

\section{Corresponding Author}

Yusuf Akcan, M.D.

Denizevler Mah

Daggülü sok No: 11/2

Samsun, Turkey

Fax: $+90-362-4353488$

E-mail: yusuf@doctor4u.com 